\title{
Learning from Experience in Positioning New Computer Products
}

\author{
THEODORE MODIS
}

\begin{abstract}
The correct positioning of new computer products has become crucially important as markets saturate and competition intensifies. The logistic function can provide an aid to product positioning. The method presented here addresses questions of price and performance only, and involves determination of learning curves from data on past successful product launches. It assumes that companies learn like individuals and that variables such as performance/price grow according to logistic curves limited by the basic technologies at hand.

Digital's experience shows that its VAX family of computers is amenable to such an analysis, which also provides insights on the overall evolution of that technology. Besides offering guidelines for product positioning, this approach provides a means for estimating price drops and/or performance enhancements necessitated from delays in product delivery.
\end{abstract}

\section{Introduction}

The world of business is an arena where ambitious and motivated individuals compete fiercely in order to make a profit. Competitive advantages are sought by all means. They are rarely found, however, on campuses of business schools. Successful entrepreneurs like to use their intuition in making decisions. Instead of systematic methodologies, they call on their instinct, "my tummy tells me," and those who are gifted in that way succeed more often than not.

Instinctive performance can and has gone a long way, particularly during prosperous times and conditions that permit one to dance one's way through good decisions. The difficulties show up during economic recessions, when markets start saturating, competition becomes cutthroat, and the name of the game changes from prosperity to survival. As with the performances of musicians or acrobats, heavy psychological stress results in insecurity, panic, and mistakes. I have been approached by executives recently saying, "We had no difficulty positioning our products back when the business was booming, but now that it has become of crucial importance, how can we be sure we are not making a mistake?"

This question was the motivation behind the work described here. The purpose was

THEODORE MODIS is a physicist who has carried out experiments in elementary particles for over 15 years at CERN, in Geneva, Switzerland; at Brookhaven National Laboratory, Upton, New York; and at NEVIS Laboratories, Irvington-on-Hudson, New York. He has also been on the teaching faculties of Columbia and Geneva Universities. Since 1984, he has been working as a Management Science consultant in Digital Equipment Corporation International (Europe), Geneva, Switzerland. 
to thn a method that describes systematically good past decisions. If the decisions had been successful, they must have followed some natural-selection law, for example, survival of the fittest. Competitive growth has been well modeled by the logistic function. In addition, learning, which plays an important role in the computer industry, also proceeds along S-shaped patterns.

At Digital, we have found that our successful products turned out to be sitting on logistic curves. Using projections of these curves, one can derive guidelines indicating which behavior will be coherent with the historical record. Such guidelines to decision making should help us continue doing the same thing and be less subject to errors resulting from today's stressful environment.

\section{Learning Curves}

Learning is a natural growth process that follows an S-shaped pattern with time. Whether it rigorously obeys the logistic-growth equation is subject to debate. The exponential-like bends of the logistic curve stem from the nature of multiplication (breeding), which for learning may not be obvious. A learning curve need not necessarily be symmetric. Still, Whiston [1] presents a graph showing that the vocabulary of a growing infant closely follows a logistic to reach a plateau of $\sim 2500$ words by the end of the sixth year. This limit can be thought of as the vocabulary "niche" of words used at home. Later, schooling enriches further the child's vocabulary, but this is a new process, probably following a similar type of curve to reach a higher plateau.

In recent years, Western society has been learning how to make computers. The computer industry displays typical logistic-growth patterns concerning innovation, measured in numbers of different computer models. [2]. The final limitation here comes from the fact that only a finite number of reasonably distinguishable computer models can be put forth from the set of basic technologies available. Different generations of computers can be visualized as a succession of $\mathrm{S}$ curves, each one coming to life following a new technological breakthrough.

Growth processes cascade. A new one picks up where the old one leaves off. Foster, in his book Innovation: The Attacker's Advantage [3], highlights the connection between a company's survival and its ability to pass from one $S$ curve to the next. Van der Erve goes one step further; in his book The Power of Tomorrow's Management [4], he attempts to guide top management on how to implement a timely jump between successive $S$ curves.

Beyond the qualitative approach of Foster and Van der Erve, however, $\mathbf{S}$ curves can be exploited quantitatively. They can be determined mathematically before they are completed by fitting the historical data to the logistic function:

$$
P(t)=\frac{M}{1+e^{-\alpha\left(t-t_{0}\right)}}
$$

Once the three constants, $M, \alpha$, and $t_{0}$, have been determined, the curve can be projected to completion. A forecast obtained in this way claims validity because niches in nature do not remain partially filled for natural reasons. Natural reasons are all conditions that existed during the historical period. For the growth of product sales, such conditions include unpredictable but not unusual events like strikes, stock market plunges, price wars, and, in general, competitors' adverse reactions.

When fitting procedures are employed to estimate the final ceiling from historical data that cover only the beginning of the process, the errors can be large. It is possible 
to evaluate these uncertainties [5], and even when they are very large, the findings may and still be illuminating.

\section{Positioning New Computer Models}

The history of successful products has been shaped by competitive growth and learning, both of which are natural processes amenable to logistic description. The first gives rise to a product's life cycle and has been exploited in forecasting sales [6]. The second describes the diffusion of the product's new technology. Technology diffusion and innovation also follow S curves [7]. Consequently, the variable that best describes the evolution of the product's characteristics over time can itself be expected to follow similar curves.

What is generally used for product positioning is the performance-price ratio. If a natural relationship exists between performance, price, and time, it should reflect the equilibrium between productivity and competition. The research engineers of each manufacturer try to produce better products than the competition. They often do. Exceptional individuals that they are, sometimes they do more than that. They may produce products that are too good by being ahead of their time. Performance-price must increase for every new model, but success, which translates to maximal customer acceptance, is linked to how accurately this increase matches customer needs. The curve traced by successful product launches does not only reflect the rate at which engineers learn how to build powerful computers. The selection process is also affected by the rate at which customers are able to assimilate the available performance.

Computer manufacturers carry out research and development programs in relative isolation in the hope of acquiring a competitive advantage. In so doing, each is going through a learning process. At the same time, different market segments absorb computing power at different rates. Consequently, one needs many curves to cover the full range of products and vendors.

Performance of computers is often measured in millions of instructions per seconds (MIPS), and the ratio MIPS/\$ becomes the usual measure of a product's performance/ price. This may not be the most crucial characteristic in the eyes of the customer, but it is one quantitative measure of competitiveness. The example treated below is Digital's VAX family of products from the very first model, the VAX 11/780, to the recent mainframes.

\section{Digital's Experience}

Figure 1 shows the historical positioning of VAX in the variable MIPS/\$, where \$ represents the selling price of the model in question expressed in millions of dollars. Use is made of the logistic transformation:

$$
\log \frac{f}{1-f} \quad \text { where } f \text { is the fraction } \quad \frac{P(t)}{M} \quad \text { from eq. } 1
$$

In this transformation, $\mathrm{S}$ curves become straight lines. The four straight lines shown are logistic fits to the corresponding data points.

Four independent curves were fit to the data. The fit parameters can be found in Table 1. The leftmost curve represents the low end, personal computers and workstations. The one on the extreme right is Digital's high end, the superminis. Estimating the final ceiling from such early sections of $\mathbf{S}$ curves implies large uncertainties on the final maximums. Our a priori knowledge, however, of a level for these ceilings is so poor 


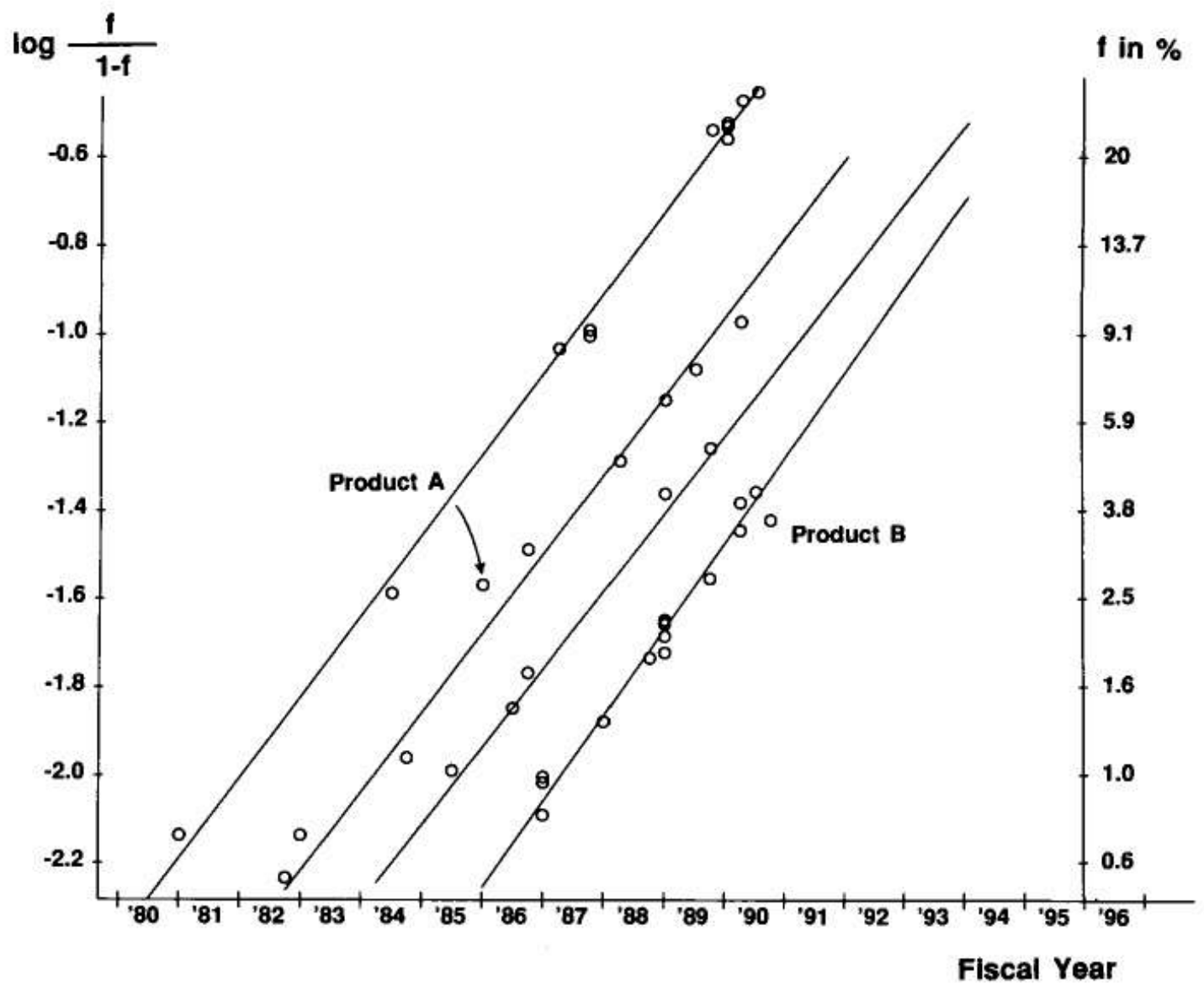

Fig. 1. Logistic fits to the data of MIPS/ $\$$ for Digital VAX products. The fraction $f$ represents the percent penetration of the niche. The very first product VAX 11/780 is not shown as it falls far below the $1 \%$ level.

that even very uncertain estimates are revealing. The ceiling was arbitrarily constrained to be the same for all four curves on the argument that the limiting factors come from the basic technologies at hand and not from the way one packages them. The following conclusions depend little on the ceiling's uncertainty:

- The low end has penetrated the performance/price niche further than the high end by a factor of 4 to 5 .

- Over the whole range Digital is far from saturating performance per dollar. On the contrary, the computer power customers will be getting for their dollar will keep increasing at an increasing rate for another five years at the low end and up to ten years at the high end.

TABLE 1

Fit Parameters for the Four Logistic Curves of Figure 1, from Left to Right

\begin{tabular}{lcccc}
\hline$M$ & 690 & 690 & 690 & 690 \\
$\alpha$ & -0.106 & -0.104 & -0.102 & -0.113 \\
$t_{0}$ & 63.61 & 73.20 & 79.67 & 81.97 \\
Correlation & 0.9984 & 0.9939 & 0.9983 & 0.9872 \\
$\begin{array}{l}\text { Mean absolute } \\
\text { error in \% }\end{array}$ & 2.83 & 2.57 & 1.61 & 2.15 \\
\hline
\end{tabular}

The time units are trimesters starting with fiscal year 1977. The ceiling $M$ was constrained to be the same for all four curves. Its determination carries an uncertainty of $\pm 60 \%$ with $90 \%$ confidence level. 
- The emergence of four coherent groups is evidence for a natural segmentation of the product range. This reflects Digital's internal structure of four business units over this historical period.

Individual products can be seen to fluctuate around the fitted patterns. These deviations, whenever significant, can be illuminating. For example, a popular model launched in 1985, designated in Figure 1 as product $A$, is seen to be to the left of the closest curve. This can mean a number of things:

- The product was announced six months too early.

- The product was too powerful by $15 \%$.

- The product was underpriced by $15 \%$.

- The product was positioned to gain market share.

It is generally agreed today that this product was positioned for market share.

Another case is the more recent product B. It falls to the right of the high-end curve as if it comes to market 4-6 months too late or is underperforming and/or overpriced. It turns out that this model is specially designed to be fault tolerant and is priced accordingly. MIPS/\$ is a crude measure of performance and will be inappropriate for positioning specialized products. For the majority of the models, however, the curves make sense and can offer guidelines in positioning future products.

Positioning products to a large extent means deciding on their performance, price, and announcement date. These three variables are interrelated according to eq. 1 :

$$
\frac{\text { MIPS }}{\text { price }}=\frac{M}{1+e^{-\alpha\left(t-t_{0}\right)}}
$$

With $M, \alpha$, and $t_{0}$ known, knowledge of two variables dictates a value for the third. The most classical application is pricing a new product. Its performance (MIPS) has been measured and is not easy to change. For a chosen announcement date, the curve gives a MIPS $/ \$$ ratio that yields a price value.

\section{Corrective Actions}

With the logistic function determined, partial derivatives can provide us with additional handles. We can obtain relationships between two of the three variables by fixing the third at a desired value. This allows us to answer questions such as, How should the price depend on the performance? How should changes in the announcement date affect the price? What performance would be correct for such a date?

A typical application is sizing the effect of sliding from schedule on product delivery. Product announcements precede shipments by a variable amount of time. Products coming to market late are worth less. How much should their price be dropped following a nonscheduled delay?

With performance (MIPS) fixed, eq. 2 can be differentiated for the price variable to give

$$
\frac{d(\text { price })}{d t}=-\frac{\alpha \cdot \text { MIPS }}{M} e^{-\alpha\left(t-t_{0}\right)}
$$




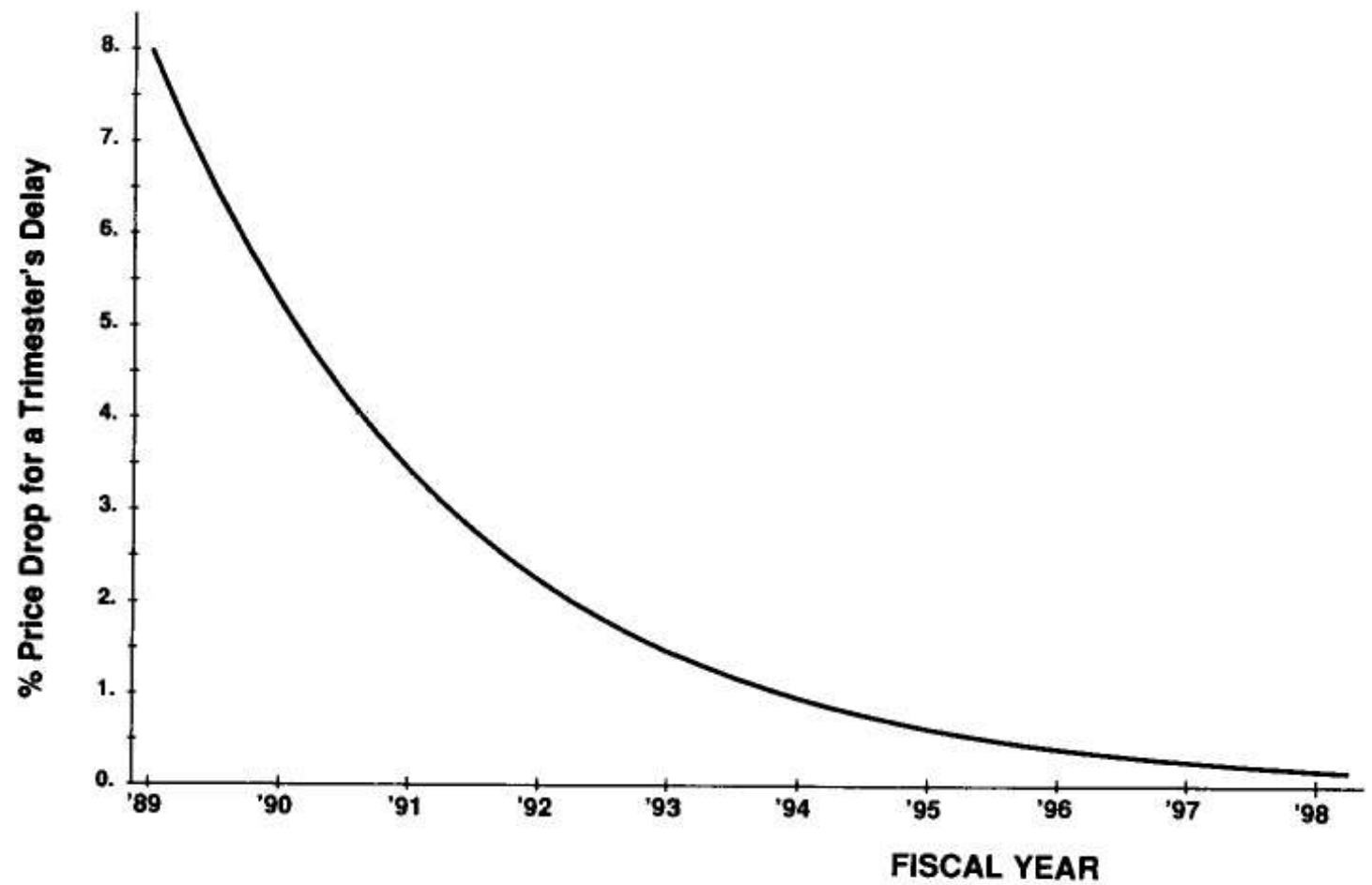

Fig. 2. Guidelines for decreasing price following delays in dellveries for products entering in the low-end range. The performance remains unchanged.

indicating that price has to decrease as shipments get delayed. Moreover, because this decrease is an exponential decay, it has a most dramatic effect in the beginning. Equation 3 is plotted in Figure 2 for the low-end group of Digital products. For a product introduced in this market segment in 1989 , a delay by one trimester required an $8 \%$ price drop; a year later, a $5.5 \%$ price drop sufficed. The graph eventually goes to zero, implying that beyond a certain time, delays will no longer warrant significant price drops. How can that be?

\section{ECONOMIES OF SCALE}

We are all familiar with the concept of economies of scale. The volume curve tells how production costs per unit decrease with the number of units produced, down to a value beyond which no further reduction is possible. The curve very much resembles an exponential decay toward a final asymptote. Cost reduction is achieved in part from automation and distribution of fixed costs over a large number of units. The most important reason for cost reduction, however, is learning. Producing large number of units results in learning, which translates to reducing costs by becoming more efficient. The volume curve is often referred to as the industry learning curve.

The inverse of eq. 2 describes costs per unit of performance, something very similar to the economies of scale:

$$
\frac{\text { price }}{\text { MIPS }}=\frac{1}{M}+\frac{e^{-\alpha\left(t-t_{0}\right)}}{M}
$$

Economies of scale say the more you produce, the less it costs per unit. Equation 4 says the longer you produce, the less it costs per MIPS. They essentially say the same thing because volume and time are correlated. 


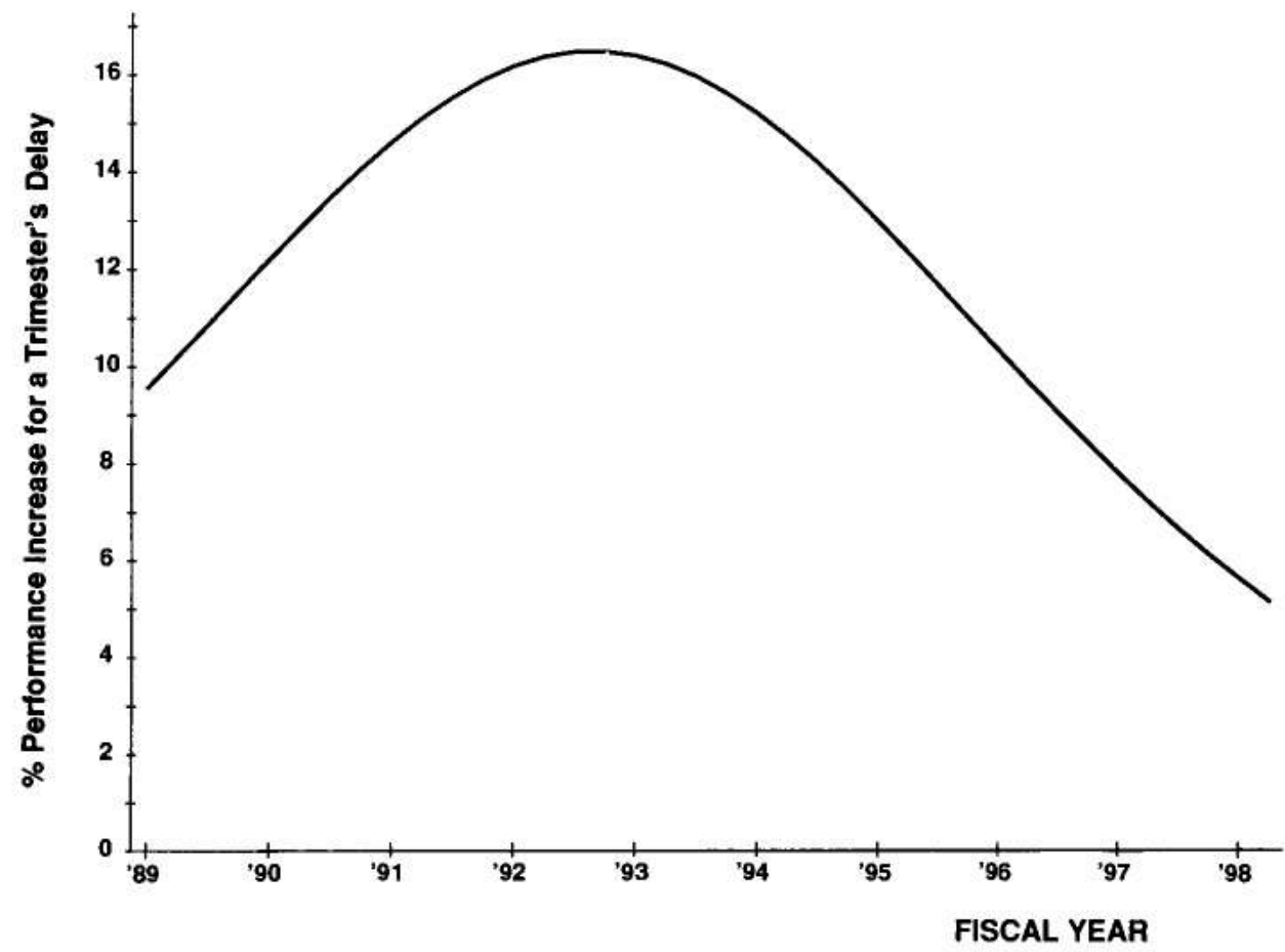

Fig. 3. Guidelines for performance adjustments following delays in delivery for products entering in the low-end range. The price remains unchanged.

The methodology exposed here links in a systematic way learning in the academic sense-logistic curves-with industrial learning. It offers a theoretical explanation for the shape of the volume curve otherwise known from practical experience only.

Figure 2 depicts the time derivative of eq. 4 , a simple exponential decay.

Another surprise waits for us in the case where performance can be adjusted, but price remains fixed. We need to calculate the required performance improvement, following a delay, in order to maintain the same price. In this case, price is fixed and eq. 2 can be differentiated for the performance variable:

$$
\frac{d(\text { MIPS })}{d(\text { time })}=\frac{\alpha \cdot M \cdot \text { price }}{\left(1+e^{\alpha\left(t-t_{0}\right.}\right)\left(1+e^{-\alpha\left(t-t_{0}\right.}\right)}
$$

This form is bell shaped. Figure 3 plots this expression for Digital's low-end market segment. It is growing for small $t$, it reaches a maximum at $t$ equals $t_{0}$, and it gets smaller for larger $t$. What does this signify?

Performance has to increase as a consequence of delayed delivery if price is to be left the same. This increase has to be larger every year as we approach $t_{0}$, the middle point in the technology learning curve, but beyond that time it starts getting smaller. Slippage in shipping a product will require less and less improvement in its performance after 1993. For a product launched in 1989, a trimester's delay required a $10 \%$ enhancement in performance in order to keep the price unchanged. This increase grows to more than $16 \%$ in 1993 , but drops back to $10 \%$ by 1996 . This can be understood in terms of 
the growth curve starting to flatten out after 1993 and consequently slippage in schedule, requiring a smaller improvement in performance than previously.

\section{New Technologies}

The first time we used this method to be guided in positioning products, we were also confronted with the introduction of the new technology reduced instruction set computing (RISC). Digital had two new powerful workstations to price, one of the traditional architecture and one of RISC architecture.

It was straightforward to recommend a price for the traditional system. The new technology product, however, posed several problems. First, the variable MIPS/\$ was no longer "kosher." A MIPS in the traditional environment is not the same as a MIPS in the RISC environment. Moreover, the experts would not be unanimous about the equivalence between the two environments. What was worse was that the appearance of RISC was something significantly more important than previous technological improvements. Would RISC follow the same learning curves or would it start its own with different parameters?

The experts were convoked for brainstorming. Besides their opinion on the equivalence of MIPS, we wanted their opinion on the following question: How different is the new technology from the old? To quantify their answers, we had a follow-up question: Is it as different as passing from tube amplifiers to transistors?

This time the experts were unanimous. RISC is a technology very different from the old technology, but not as different as transistors were to tubes. From then onward it was a question of judgment. For lack of other guidelines the final recommendation was to put RISC on the low-end curve and raise the flag of caution at the same time.

A few trimesters later, the market price of both products turned out to be close to the recommendations. It still remains a possibility, however, that RISC will evolve according to completely different curves. Evidence based on only a few products indicates that the new technology will reach significantly higher ceilings.

\section{Conclusions}

The curves of Figure 1 on linear scales look very much like exponentials as they occupy the very early range of S-curves. In fact, had product positioning been our only concern, simple exponential functions would have sufficed. It is the long-term trends that would have been wrong in the exponential picture. We would have obtained no insight for the penetration into this technology and would have missed altogether the intriguing phenomenon mentioned earlier-the eventual decrease in performance improvements necessitated from delays.

The old technologies saturate as we enter the 21 st century. New growth curves should appear at some time. As mentioned earlier, logistic curves cascade, and the RISC technology is well poised for providing the new wave.

Confidence in positioning products via determination of logistic curves stems from the fact that such curves describe natural growth processes from beginning to end. If an early window of such a process witnesses success for whatever reasons, its natural projection should also be successful for the same reasons. Guidelines obtained in this way can serve as lighthouses through the storm of recession, price wars, cutthroat competition, and panic.

\section{References}

1. Whiston, T. G., Life is Logarithmic, in Advances in Cybernetics and Systems, J. Rose, ed., Gordon and Breach, London, pp. 265-298. 
2. Modis, T., and Debecker, A., Innovation in the Computer Industry, Technological Forecasting and Social Change 33, 267-278 (1088).

3. Foster, R., Innovation: The Attacker's Advantage, Macmillan, New York, 1986, Ch. 4.

4. Van der Erve, M., The Power of Tomorrow's Management, Heinemann, London, 1989, Ch. 7.

5. Debeceker, A., and Modis, T., Determination of the Uncertainties in S-curve Logistic Fits, International Conference on Diffusion of Technologies and Social Behavior, 14-16 June 1989, Laxenburg, Austria.

6. Day, G., The Product Life Cycle: Analysis and Applications Issues, Journal of Marketing 45, 60-67 (1981).

7. Mahajan, V., Muller, E., and Bass, F.-M., New Product Diffusion Models in Marketing: A Review and Directions for Research, Journal of Marketing 54, 1-26 (1990). 